\title{
Identification of a Novel Point Mutation of Mouse Atp2b2 Induced by $N$-Ethyl- $N$-Nitrosourea Mutagenesis
}

\author{
Lin $\mathrm{XU}^{1)}$, Zixing $\mathrm{WANG}^{2)}$, Xiwen $\mathrm{XIONG}^{2)}$, Xingxing $\mathrm{GU}^{2)}$, Xiang $\mathrm{GAO}^{2)}$, and Xia GAO ${ }^{1)}$ \\ ${ }^{1)}$ Department of Otolaryngology and Head \& Neck Surgery, The Affiliated Drum Tower Hospital of \\ Nanjing University Medical School, Nanjing 210008 and ${ }^{2}$ MOE Key Laboratory of Model Animal for \\ Disease Study, Model Animal Research Center, Nanjing University, Nanjing 210061, China
}

\begin{abstract}
N}$-ethyl- $\mathrm{N}$-nitrosourea (ENU)-induced mutagenesis is an important approach in the study of gene function and the establishment of human disease models. Here we report an ENU-induced mutation, Elfin, as a mouse model with hearing loss. Homozygous mutants were deaf and displayed severe ataxia, while heterozygous mice had a significant hearing loss. Histological analysis of the inner ear revealed that Elfin had progressive degeneration of the organ of Corti, spiral ganglion cells and an absence of otoconia in the vestibular system. The new mutation was mapped to chromosome 6 between microsatellite markers D6Mit39 and D6Mit254, where the $\mathrm{Ca}^{2+}$-ATPase type 2 (Atp2b2) gene resides. Sequence analysis revealed a unique T-to-A transition mutation at amino acid 655 resulting in lle-to-Asn substitution. These results for the Elfin mutant confirm the role of ATP2B2 in balance, hearing and formation of otoconia and suggest it may serve as a new model of human hereditary hearing loss.
\end{abstract}

Key words: Atp2b2, auditory brainstem responses (ABR), cochlea, Elfin, ENU mutagenesis

\section{Introduction}

Hearing impairment occurs in approximately 1 in 1,000 human individuals and about $2.3 \%$ of them suffer progressive hearing loss (presbycusis). It is estimated that up to $1 \%$ of the approximately 30,000 or more human genes are required for normal hearing function [7]. In general, genetic hearing loss can be divided into syndromic hearing loss (SHL) that is associated with a variety of other anomalies, and nonsyndromic hearing loss (NSHL) in which hearing loss is a unique manifestation or the only disorder. NSHL is mainly caused by inner ear defects, while most cases of SHL are external and/ or middle ear developmental defects. About one half of NSHL cases are due to monogenic forms of hearing loss and more than 80 loci have been mapped to the human genome [21, 22]. However, the precise mutations of most NSHL cases remain unknown.

The plasma membrane $\mathrm{Ca}^{2+}$-ATPase family is essential for maintaining an appropriate concentration of intracellular $\mathrm{Ca}^{2+}$ controlling $\mathrm{Ca}^{2+}$ extraction from the cell. The plasma membrane ATPase family of $\mathrm{Ca}^{2+}$ transporters consists of four subtypes, ATP2B 1-4, which are encoded by distinct genes, Atp2bl-4 (also known as Pmcal4) $[3,17]$. Of the four basic subtypes of the plasma membrane $\mathrm{Ca}^{2+}$-ATPase pump, ATP2B1 and ATP2B4

(Received 19 February 2010 / Accepted 24 September 2010)

Address corresponding: X. Gao, Model Animal Research Center, 12 Xuefu Road, Pukou District, Nanjing 210061, China. X. Gao, Department of Otolaryngology, the affiliated Drum Tower Hospital of Nanjing University Medical School, 321 Zhongshan Road, Gulou District, Nanjing 210008, China. 
have been shown to be broadly expressed, and are often referred to as housekeeping genes, whereas ATP2B2 and ATP2B3 are localized in specialized tissues, primarily in the brain [14]. Notably, several studies have detected Atp2b2 expression in the inner ear $[5,16,24]$. In the cochlea, ATP2B2 is localized in both inner and outer hair cell stereocilia, as well as in spiral ganglion neurons [5, $16,24]$. In addition, ATP2B 2 has also been found to be expressed in vestibular hair cells $[16,24]$. It is significant that, ATP2B2 is believed to be the only $\mathrm{Ca}^{2+}$ transporter in the stereocilia, indicating that it plays a critical role in maintaining $\mathrm{Ca}^{2+}$ homeostasis during hair cell transduction. Indeed, the deafwaddler $(d w f)$ mice with an Atp2b2 mutation are deaf and display vestibular imbalance, which further demonstrates that ATP2B2 function is critical for both hearing and balance [16].

Because the auditory systems of mice and humans are conserved, mouse models are usually adopted for studying the pathogenesis of human hearing loss. $\mathrm{N}$-ethyl- $\mathrm{N}$ nitrosourea (ENU) is a powerful chemical mutagen that offers the possibility of inducing genome-wide point mutations at high frequency [1]. ENU-induced mutagenesis has recently become a powerful tool for investigating gene functions and generating of human disease models [2]. In this study, we report the identification of a novel ENU-induced mouse mutant, Elfin, as an animal model of human hearing loss. Elfin mice exhibit age-related hearing impairment with progressive degeneration of the cochlea structure as well as reduction in spiral ganglion cell numbers. Mapping and sequencing analysis revealed that the Elfin mutation is a T-to-A missense transition at nucleotide 1964 in the Atp2b2 coding sequence that results in Ile-to-Asn substitution at amino acid 655 .

\section{Materials and Methods}

\section{Mice and ENU mutagenesis}

The institutional animal care and use committee (IACUC) of the Model Animal Research Center of Nanjing University approved all animal procedures. The $\mathrm{C} 57 \mathrm{BL} / 6 \mathrm{~J}$ and $\mathrm{C} 3 \mathrm{H} / \mathrm{HeJ}$ mice were maintained on a $12 \mathrm{~h}$ light/12h dark cycle under specific pathogen-free conditions. The Elfin mutation was generated in an ENU mutagenesis program using $\mathrm{C} 57 \mathrm{BL} / 6 \mathrm{~J}$ mice, which is being performed as previously described $[8,23]$.

\section{Mutation mapping and cloning}

Elfin/+ males were mated to $\mathrm{C} 3 \mathrm{H} / \mathrm{HeJ}$ females to generate $\mathrm{F}_{1}$ mice. $\mathrm{F}_{1}$ males with auditory defect were backcrossed to $\mathrm{C} 3 \mathrm{H} / \mathrm{HeJ}$ female mice to generate $\mathrm{N}_{2}$. To narrow the genetic interval harboring the mutation, DNA was obtained from the tails of 42 mutant $\mathrm{N}_{2}$ mice. Polymorphic microsatellite markers from the Whitehead/MIT database (http://www.broad.mit.edu) were used for PCR assays. To examine whether the mutation resides in the Atp2b2 gene, total RNA samples were prepared from the brain of adult male C57BL/6J and Elfin/Elfin mice using a Trizol kit, following the manufacturer's instructions (Shenergy, Shanghai, China). Full length cDNA of At$p 2 b 2$ was synthesized with AMV reverse transcriptase (Promega, Madison, WI, USA). Due to the large size of Atp $2 b 2$ cDNA, four overlapping cDNA fragments that covered the full coding region were amplified by PCR with Pyrobest Taq polymerase (TaKaRa, Dalian, China) using the following primers:

Atp2b2-1-F: 5'-GCCCAGCCTGAGTAGACACTTC-3'; Atp2b2-1-R: 5'-CACTCTTCAGAGGCTGCATCTC-3'; Atp2b2-2-F: 5'-ATATGGTGACCTTCTTCCCGC-3'; Atp2b2-2-R: 5'-TGCCTCAAGTCCAGCACAAAG-3'; Atp2b2-3-F: 5'-TCTTCATCATTGGTGTGA-3'; Atp2b2-3-R: 5'-CCTTGACGATGCTGCTGAAG-3'; Atp2b2-4-F: 5'-GATGACAACTTCAGCAGCATC-3'; Atp2b2-4-R: 5'-TCAGCTAAAGCGACGTCTCCAG-3' .

The four resulting fragments were then subcloned into TA cloning vector pMD-18T (TaKaRa) and sequenced (Bioasia, Shanghai, China). All sequences were confirmed by sequencing from both directions.

\section{Auditory brainstem responses}

Before ABR measurements, mice were anesthetized by intraperitoneal injection with Avertin (Sigma Aldrich, St. Louis, MO, USA) at $0.5 \mathrm{~g} / \mathrm{Kg}$ body weight. Three electrodes, one under each ear and one at the top of the skull, were inserted subcutaneously. Specific auditory stimuli (broad-band click and pure-tone pips of 8, 16, and $32 \mathrm{kHz}$ ) from calibrated sound transducers were delivered binaurally through plastic tubes to the ear canals. ABR responses were recorded with a PowerLab recorder (ADInstruments, Sydney, Australia). The threshold of hearing was determined by observing the lowest intensity of sound required to elicit a character- 
istic waveform. The ABR test was repeated twice for each confirmed mutant mouse.

\section{Morphologic analysis of the inner ear}

Mice were perfused with phosphate buffer followed by $4 \%$ paraformaldehyde (Sigma Aldrich) solution. The cochleae were removed and stored in $4 \%$ paraformaldehyde at $4^{\circ} \mathrm{C}$ overnight. For decalcification, the cochleae were incubated in $10 \%$ EDTA ( $\mathrm{pH} 7.4$ ) for 7 days at $4^{\circ} \mathrm{C}$ before embedding in paraffin. Serial sections $(7 \mu \mathrm{m})$ of the cochleae were stained with hematoxylin and eosin (HE).

\section{Transmission electron microscopy (TEM) assay}

After perfusion, cochleae from mice with different genotypes (Elfin/+ and $+/+$ ) were further fixed in $2.5 \%$ glutaraldehyde (Sigma Aldrich) in $0.15 \mathrm{M}$ sodium cacodylate (Sigma Aldrich) buffer at $4^{\circ} \mathrm{C}$ overnight. After washing in sodium cacodylate buffer, cochleae were decalcified with $10 \%$ EDTA (pH 7.4) at $4^{\circ} \mathrm{C}$ for 7 days. Samples were then post-fixed in $1 \%$ osmium tetroxide in phosphate buffer, dehydrated in graded ethanol and embedded in epoxy resin. Sections, $90 \mathrm{~nm}$ thick, were mounted on copper grids, stained with uranyl acetate and lead citrate, and examined by a Hitachi H7100 TEM (Hitachi High-Technologies, Tokyo, Japan).

\section{Statistical analysis}

Data are presented as mean \pm SEM, and statistical analysis was performed using the unpaired Student's $t$-test. Differences with $P<0.05$ were considered to be statistically significant.

\section{Results}

\section{Progressive hearing loss in Elfin mutant mice}

In the course of primary auditory screen of ENUmutated C57BL/6J mice, we recorded auditory brainstem response (ABR) to click-sound (a mixed range of lower frequencies, mostly between 2 and $10 \mathrm{kHz}$ ) stimuli. Interestingly, a male mouse of the $\mathrm{G}_{1}$ generation was identified with severe hearing loss. The affected male mouse gave rise to affected male and female progeny after mating with wild-type C57BL/6J females, indicating that this heritable mutation was autosomal dominant. De-

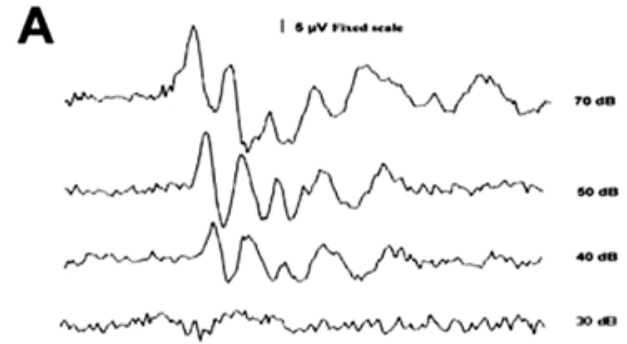

B

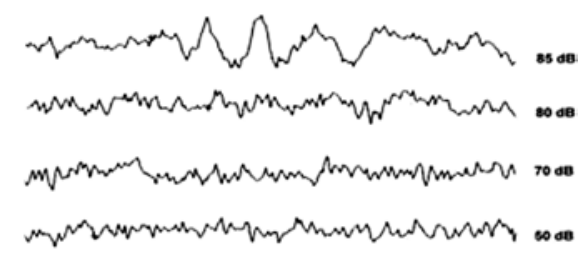

C

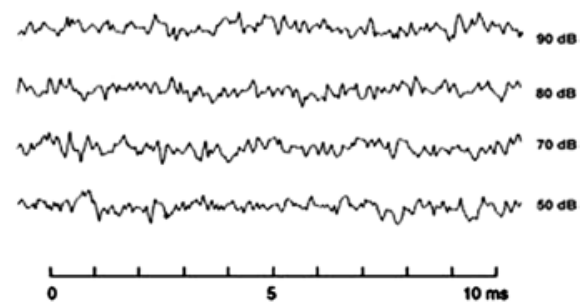

D

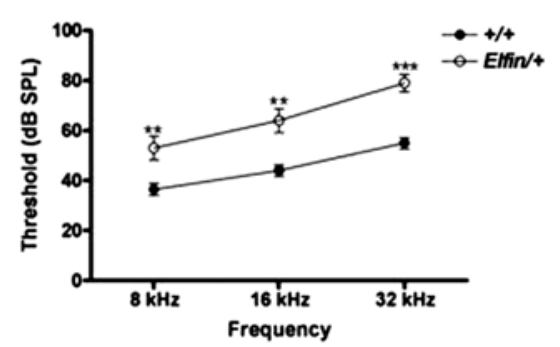

Fig. 1. Auditory brainstem response analysis of Elfin mice. (A, B) ABR recordings from (A) 2-week-old and (B) 4-week-old Elfin/+ mice after exposure to click stimuli. (C) ABR recordings from 3-week-old Elfin/Elfin mice after exposure to click stimuli. (D) ABR thresholds of Elfin/+ mice and +/+ mice in response to different frequencies of sound stimuli: 8,16 , and $32 \mathrm{kHz}$ ( $\mathrm{n}=10$ per group). Data shown are mean \pm SEM. $* * P<0.01$, $* * * P<0.001$.

tailed ABR measurements with click stimuli were carried out to evaluate the hearing thresholds of Elfin/+ mice at different ages. Elfin/+ mice demonstrated a characteristic $\mathrm{ABR}$ waveform, beginning at sound pressure levels of $40 \mathrm{~dB}$ at 2 weeks of age (Fig. 1A). At 4 weeks of age, Elfin/+ mice displayed a significantly elevated ABR 
threshold of up to $85 \mathrm{~dB}$ SPL (Fig. 1B). At 3 months, none of the Elfin/+ mice exhibited a detectable waveform even at $90 \mathrm{~dB}$ SPL, indicating that the Elfin/+ mice were completely deaf (data not shown). In addition, we also examined the hearing threshold of mice homozygous for Elfin. As expected, no waveform was observed in 3-week-old Elfin/Elfin mice even at sound stimuli of 90 dB SPL (Fig. 1C). To further assess auditory system function, average thresholds were determined with sound stimuli of pure tones of 8,16 , and $32 \mathrm{kHz}$ delivered to 4-week-old Elfin/+ mice and wild-type mice. As shown in Fig. 1D, Elfin/+ mice showed markedly elevated thresholds for each of the 3 sound stimuli $(53 \pm 4.67 \mathrm{~dB}$ at $8 \mathrm{kHz}, 64 \pm 4.76 \mathrm{~dB}$ at $16 \mathrm{kHz}$, and $79 \pm 3.48 \mathrm{~dB}$ at $32 \mathrm{kHz}$ ), when compared with the thresholds of wildtype controls $(36.5 \pm 2.48 \mathrm{~dB}$ at $8 \mathrm{kHz}, 44 \pm 2.33 \mathrm{~dB}$ at $16 \mathrm{kHz}$, and $55 \pm 2.24 \mathrm{~dB}$ at $32 \mathrm{kHz}$ ). These results demonstrate that Elfin/+ mice have a severe and agerelated progressive hearing loss.

\section{Histology of the cochlea structure of Elfin mutant mice}

Light microscopic analysis of cochlea sections showed normal architecture of the organ of Corti with well formed stria vascularies and spiral ganglion cells in 2-month-old Elfin/+ mice, even though these mice displayed impaired hearing function (Fig. 2A). In contrast, electron microscopic assays of Elfin/+ mutants at 2 months revealed the degeneration of outer hair cells $(\mathrm{OHC})$. Condensed chromatins and decreased organelles were observed in $\mathrm{OHC}$ of Elfin/+ mice, indicating that the $\mathrm{OHC}$ were undergoing apoptosis (Fig. 2E). In addition, disruption of the dense plate of $\mathrm{OHC}$ at the base of stereocilia was also observed in $\mathrm{OHC}$ of Elfin/+ mice (Fig. 2E). In 4-month-old Elfin/+ mutants, degeneration of the organ of Corti and spiral ganglia was obvious in the basal turn of the cochlea (Fig. 2C), but the severity of degeneration decreased towards the apex (Fig. 2D). Similar to the histological defects of the cochlea observed in 4-month-old Elfin/+ mice, severe degeneration of the organ of Corti was identified in a younger group of homozygous Elfin mutants at 3 weeks of age (Fig. 2B).

\section{Ataxia in homozygous Elfin mutants}

About $25 \%$ of mice derived from Elfin/+ intercross
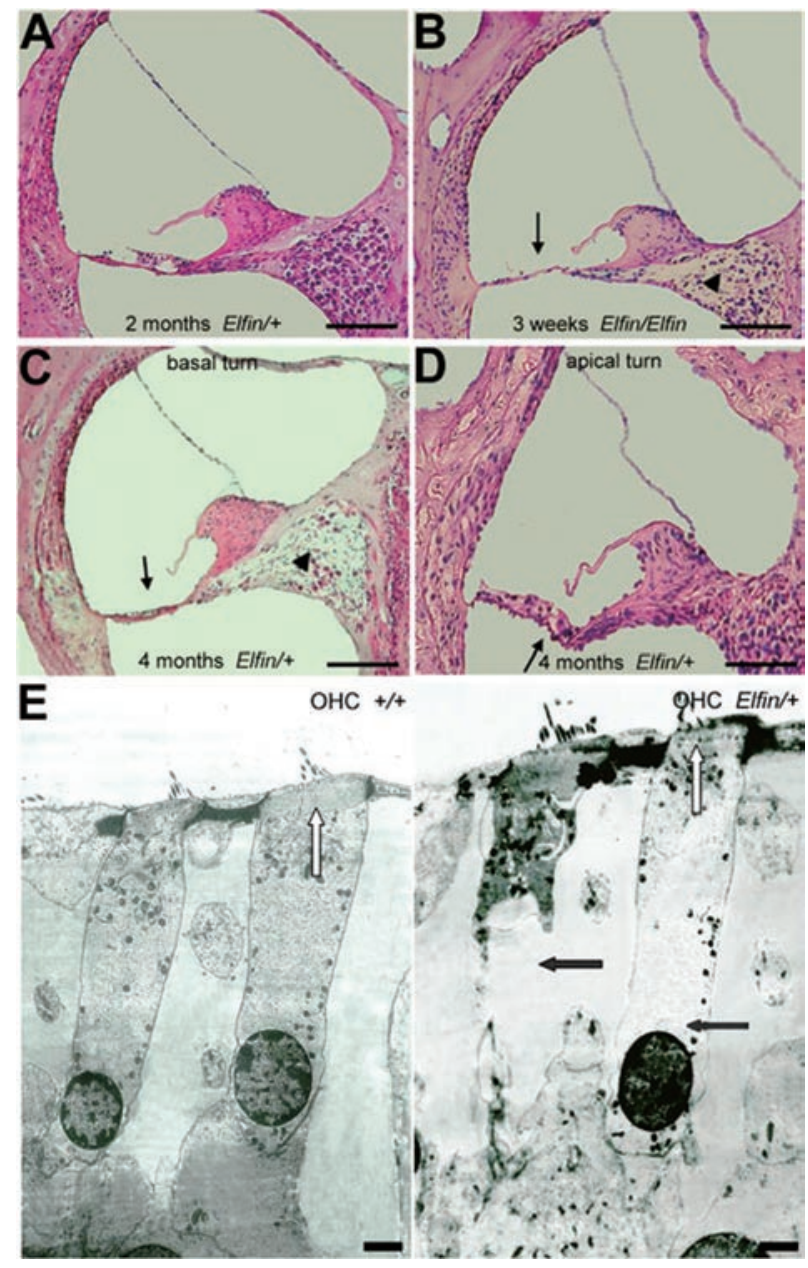

Fig. 2. Histology of the cochlea in Elfin mice. (A-D) HE stained sections of the cochleae of Elfin/+ mice. (A) Cochlear structure appears normal in 2-month-old Elfin/+ mice. (B) Degeneration of the organ of Corti (arrow) and spiral ganglion cells (arrow head) is apparent in 3-week-old Elfin/ Elfin mice. (C, D) Degeneration of the organ of Corti (arrow) and spiral ganglion cells (arrow head) is apparent in the basal turn of the cochlea (C), and to a lesser extent in the apical turn of the cochlea (D) in 4-month-old Elfin/+ mice. Scale bar, $100 \mu \mathrm{m}$ (A-D). (E) Electron micrographs of the organ of Corti in 2-month-old mice show the normal appearance of $\mathrm{OHC}$ and cuticular plate (white arrow) in wild-type mice compared with the damaged OHC (black arrow) and the abnormal cuticular plate (white arrow) in Elfin/+ mice. Scale bar, $5 \mu \mathrm{m}(\mathrm{E})$.

exhibited severe ataxia and reduced body weight ( 20\%) compared with their wild-type or heterozygous littermates. These mice were considered to be homozygous mutants and were later confirmed as such by molecular genotyping. Elfin/Elfin mice had great difficulty in main- 


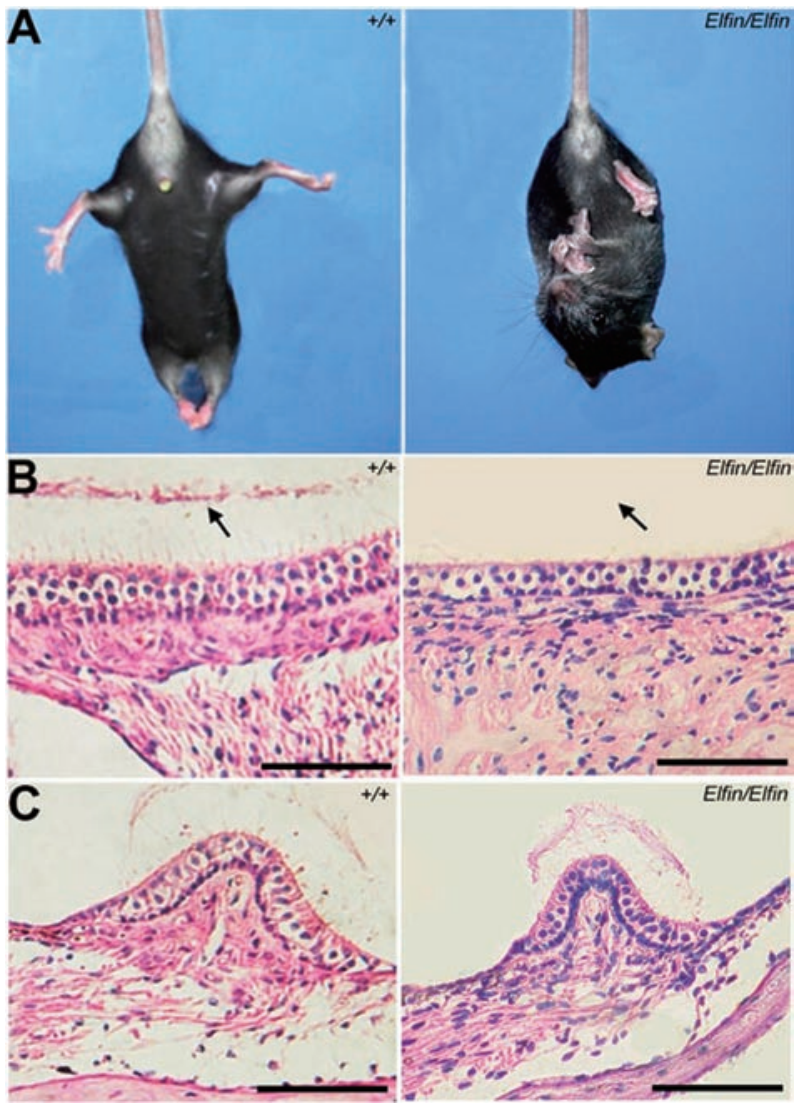

Fig. 3. Histology of the vestibular system in Elfin/Elfin mice with balance deficit. (A) Views of 6-week-old mice lifted by tail. The wild-type mice showed normal balance behavior, whereas the Elfin/Elfin mice clenched their rear legs and feet together when lifted by tail. (B) HE stained sections of the saccules of 2-month-old wild-type and Elfin/Elfin mice. Otoconia, calcium carbonate crystals embedded in the otolithic membrane, are absent in Elfin/Elfin mice. (C) HE stained sections of the crista ampullaris of 2-month-old wild-type and Elfin/Elfin mice. The structure of the crista ampullaris is histologically similar in both genotypes. Scale bar, $50 \mu \mathrm{m}$.

taining their balance when walking or standing, and always clenched their rear legs and feet together when lifted by their tail (Fig. 3A). Consistent with the ataxia phenotype, abnormal architecture of the vestibular system was observed in Elfin/Elfin mutant mice. Histological examination revealed that calcium carbonate crystals and hair cells in the saccule were missing in the vestibule of Elfin/Elfin mice (Fig. 3B). However, hair cells, support cells, and innervation of the sensory epithelium in the utricle and saccule of homozygous mutants appeared normal (Fig. 3C).
Elfin is an allele with a point mutation of the ATP $2 B 2$ gene

For genetic mapping analysis of the Elfin locus, C57BL/6J background Elfin/+ mice were backcrossed to $\mathrm{C} 3 \mathrm{H} / \mathrm{HeJ}$ mice. To carry out the mutation mapping, we initially scanned the whole genome using several microsatellite markers scattered in different chromosomes. Initial genome scanning indicated significant linkage between Elfin and D6Mit74, suggesting that the Elfin mutation was located in Chromosome 6. Elfin was further mapped to a $\sim 18.8 \mathrm{Mb}$ region on mouse chromosome 6 between markers D6Mit39 and D6Mit254 (Fig. $4 \mathrm{~A})$. Among the candidate genes in this region, Atp2b2 (also named Pmca2) has been reported to be involved in hearing loss. Sequence analysis of the coding region revealed a T-to-A missense transition at nucleotide 1964 in the Atp $2 b 2$ coding sequence that results in an Ile (I) to Asn (N) substitution at amino acid 655 (Fig. 4B). This mutation is located in the largest intracellular loop between transmembrane domains 4 and 5, and Ile655 is highly conserved among the plasma membrane $\mathrm{Ca}^{2+}$ ATPase family members (Fig. 4C and 4D).

\section{Discussion}

Calcium homeostasis is fundamental to diverse cellular processes. A number of mechanisms have been developed to maintain the concentration of free $\mathrm{Ca}^{2+}$ inside cells. Plasma-membrane $\mathrm{Ca}^{2+}$-ATPase, which extracts $\mathrm{Ca}^{2+}$ from cytoplasm, is essential for maintaining low intracellular calcium in all eukaryotic cells [3]. The members of the plasma-membrane $\mathrm{Ca}^{2+}$-ATPase family are predicted to contain 10 transmembrane regions (TM). Their $\mathrm{NH}_{2}$ and $\mathrm{COOH}$ termini are both located on the cytosolic side of the membrane [4, 17]. Approximately $80 \%$ of the protein mass is facing the cytosol and consists of three major parts: the intracellular loop between transmembrane segments 2 and 3, the large unit between membrane-spanning domains 4 and 5 , and the extended "tail" following the last transmembrane domain [4, 17]. The largest cytosolic loop between TM4 and TM5 contains the major catalytic domains: P-domain and N-domain $[4,18]$. The P-domain contains the invariate aspartate residue that forms the acyl phosphate intermediate during ATP hydrolysis, whereas the N- 
A

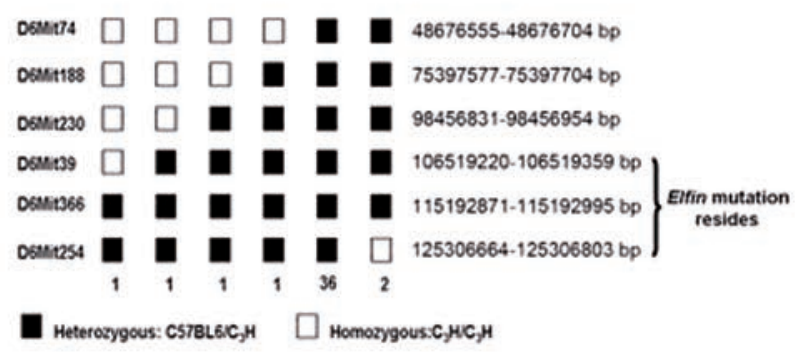

C

AIPzo

ATPSes

Arpas

AtProv

ATP283

AтP260

AтP2)

AтP2)

AIPzeA

AтP2B

AIPZB

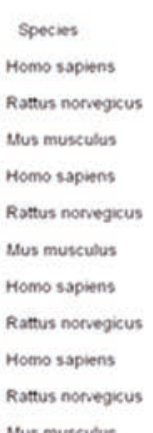

Mus musculus

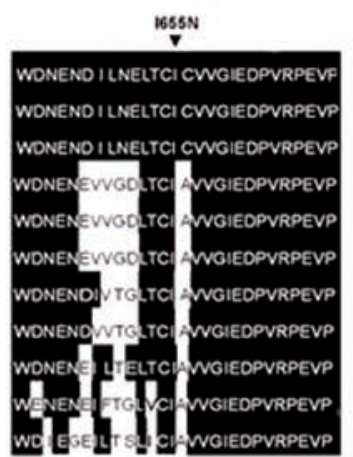

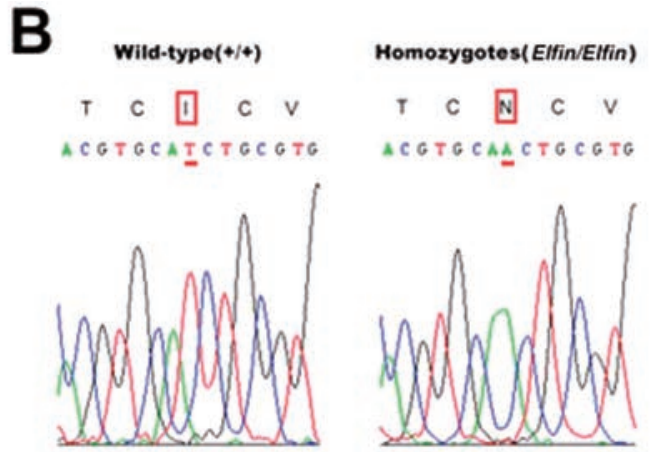

D

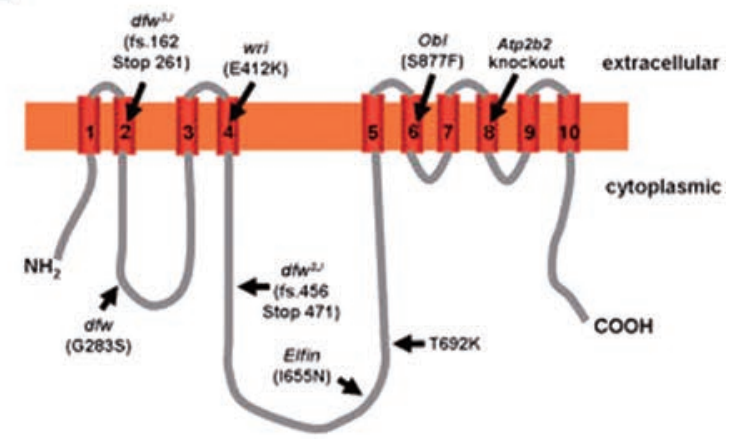

Fig. 4. Identification of Elfin mutation. (A) Allele distribution patterns of 42 Elfin/+ backcross offspring. Elfin was mapped to a $\sim 18.8$ Mb region on mouse chromosome 6 between markers D6Mit39 and D6Mit254. The physical position of each microsatellite marker in chromosome 6 is also shown. (B) Sequence of the Atp $2 b 2$ gene in Elfin/Elfin mice identifying a T-to-A transition, which leads to change of Ile to Asn at amino acid 655. (C) Sequence alignment of mouse ATP2B2 with other plasma membrane $\mathrm{Ca}^{2+}$-ATPase proteins. Arrowhead indicates the position of I655N identified in Elfin. Identical residues are highlighted with a dark background. I655 is conserved in the plasma membrane $\mathrm{Ca}^{2+}$-ATPase family protein. (D) Schematic representation of ATP2B2 with the locations of mutations. Mutations of $d f w, d f w^{2 J}, \mathrm{~T} 692 \mathrm{~K}$, and Elfin are in the intracellular loops. Mutations of $w r i, d f w^{3 J}$, and $\mathrm{Obl}$ are in the transmembrane domains. Atp $2 b 2$ knockout is the targeted null allele.

domain contains the conserved lysine which is partially involved in binding of ATP $[4,18]$. ATP2B2 exhibits a highly restricted tissue distribution, suggesting that it serves more specialized physiological functions than some of the other $\mathrm{Ca}^{2+}$-ATPase isoforms [14]. A unique role in hearing and balance is indicated by the high levels of Atp2b2 expression in the inner ear hair cells and spiral ganglion cells and this has been confirmed by our ENU-mutated Elfin mice. Elfin is a T-to-A missense transition at nucleotide 1964 in the Atp2b2 coding sequence that results in an Ile (I) to Asn (N) substitution at amino acid 655. Ile655 is highly conserved among all plasma-membrane $\mathrm{Ca}^{2+}$-ATPase isoforms and resides in the $\mathrm{N}$-domain of the largest cytosolic loop between TM4 and TM5 of ATP2B2. The Elfin mutation in the $\mathrm{N}$-domain may interfere with the domain structure for
ATP binding and result in loss of $\mathrm{Ca}^{2+}$-ATPase activity.

So far, seven mouse mutants harboring mutations in Atp $2 b 2$ have been described, including: deafwaddler (dfw) [16], deafwaddler 2J $\left(d f w^{2 J}\right)$ [16], deafwaddler 3J $\left(d f w^{3 J}\right)$ [11], wriggle mouse sagami (wri) [19], Atp2b2 null mutant [9], Oblivion $(\mathrm{Obl})$ [15] and a spontaneous T692K mutation in Atp2b2 [20]. The $d f w$ was the first mutant allele of Atp2b2 to be identified, and encompasses an A-to-G missense mutation resulting in a Glyto-Ser substitution at amino acid 283 in the intracellular loop between $\mathrm{T} 2$ and $\mathrm{T} 3$ [16]. The $d f w^{2 J}$ contains a 2-nucleotide deletion at nt1368-1369, resulting in a premature stop codon [16]. The $d f w^{3 J}$ allele encompasses a 4-nucleotide (nt483-486) deletion leading to a frame-shift and predicted truncation of ATP2B2 [11]. The wri mutation is a G-to-A transition at nt 1234 result- 
ing in a Glu-to-Lys substitution at amino acid 412 in the 4th transmembrane domain [19]. The Atp2b2 null mice were generated by inserting the neo cassette into the exon 19 of the Atp2b2 gene [9]. The $\mathrm{Obl}$ is an ENU induced mutant allele, which contains a C-to-T missense mutation resulting in a Ser-to-Phe substitution at amino acid 877 [15]. The spontaneous T692K mutation contains a Cto-A transition, causing an amino acid substitution of threonine by lysine in the cytosolic loop between $\mathrm{T} 4$ and T5 [20]. Mice heterozygous for these known mutant Atp $2 b 2$ alleles have normal movement and motor function but are severely deficient in hearing, while homozygous mutants show severe ataxia as well as profound deafness. The Elfin mutation reported here is the eighth mutation in the mouse Atp $2 b 2$ gene to be reported. Consistent with the phenotypes reported for other Atp $2 b 2$ mutants, homozygous Elfin mutants exhibit severe balance disorder, motor incoordination, and profound hearing loss. Heterozygous Elfin mice have no balance disorder but have age-related progressive hearing loss. Although one Ile to Asn alteration at amino acid 655 is the only amino acid change of the entire ATP2B2 protein, we also wonder whether this alteration is the only mutation responsible for the observed abnormalities in Elfin mice. Actually, a complementation test between Elfin and one existing Atp2b2 mutant allele would be the best way to investigate whether the Elfin mutation is a pathogenic mutation. However, we cannot perform this experiment because we have no other Atp2b2 mutant mouse strains. Nevertheless, we believe that the Atp2b2 mutation is the cause of the Elfin mice phenotypes based on not only the mapping and sequence data, but also the phenotype similarities between Elfin mice and other Atp2b2 mutants. Moreover, the Elfin mutant mice have been crossed to $\mathrm{C} 57 \mathrm{BL} / 6 \mathrm{~J}$ for more than 10 generations, therefore other mutations are unlikely associated with the Elfin phenotypes.

Atp2b2 gene dosage, as well as modest changes in ATP2B2 pumping activity is reported to produce significant phenotypic changes [11]. The $d f w$ mutation leads to a partial loss of function and ATP2B2 retains $30 \%$ of its $\mathrm{Ca}^{2+}$ pumping activity $[12,16]$. Mutations such as $\operatorname{Atp} 2 b 2^{-{ }^{--}}, d f w^{2 \mathrm{~J}}, d f w^{3 \mathrm{~J}}$, and $w r i$, are null alleles confirmed by analysis of mRNA transcripts and protein levels $[9,11,16,19]$. Previous reports have demon- strated that mice homozygous for the functional null mutations exhibit marked hearing impairment between 3 and 5 weeks of age while the $d f w$ homozygotes show no significant hearing loss [11]. Notably, mice homozygous for the null mutations display severe ataxia that prevent them from standing or walking whereas $d f w / d f w$ mice only have a wobbly gait [11]. Therefore, hearing loss and ataxia are proportional to the loss of ATP2B2 function. Elfin heterozygous mice exhibit mild hearing loss before the age of one month, which then worsens progressively with age. Elfin homozygotes are profoundly deaf and display severe ataxia. The phenotypes observed in Elfin mice are similar to those of Atp2b2 null mutants, indicating that Elfin is also a null mutant allele of Atp2b2.

Progressive hearing loss is very common in the human population, but we know little about the molecular mechanisms. Interestingly, recent reports have demonstrated that the $A T P 2 B 2$ mutation increases the severity of the progressive hearing loss seen in human patients with other gene defects, although an ATP2B2 mutation alone is not sufficient to cause hearing impairment $[6$, 10, 13]. Therefore, identifying other mutations in the Atp2b2 locus and its modifiers in mouse inbred strains may be of importance in elucidating the mechanisms involved in progressive hearing loss in humans.

In summary, we generated and identified a novel $A t$ $p 2 b 2$ mutant mouse strain with age-related hearing loss. More detailed analyses need to be performed on the Elfin mouse to elucidate the mechanism by which the I655N mutation affects auditory and vestibular function. The Elfin mouse may provide us with a new model leading to a better understanding of the structural and functional characteristics of the ATP2B2 protein.

\section{Acknowledgments}

We thank Haibin Ruan and Haibo Sha for technical help. This work was supported by the National Natural Science Foundation of China (30973302), the Medically Important People Project of Jiangsu Province (RC2007010), and the Medically Important Development Project of Nanjing (ZKX06019). 


\section{References}

1. Balling, R. 2001. ENU mutagenesis: analyzing gene function in mice. Annu. Rev. Genomics Hum. Genet. 2: 463-492.

2. Brown, S.D. and Hardisty, R.E. 2003. Mutagenesis strategies for identifying novel loci associated with disease phenotypes. Semin. Cell. Dev. Biol. 14: 19-24.

3. Carafoli, E. 1992. The $\mathrm{Ca}^{2+}$ pump of the plasma membrane. J. Biol. Chem. 267: 2115-2118.

4. Di Leva, F., Domi, T., Fedrizzi, L., Lim, D., and Carafoli, E. 2008. The plasma membrane $\mathrm{Ca}^{2+}$ ATPase of animal cells: structure, function and regulation. Arch.Biochem. Biophys. 476: 65-74.

5. Dumont, R.A., Lins, U., Filoteo, A.G., Penniston, J.T., Kachar, B., and Gillespie, P.G. 2001. Plasma membrane $\mathrm{Ca}^{2+}$-ATPase isoform 2a is the PMCA of hair bundles. $J$. Neurosci. 21: 5066-5078.

6. Ficarella, R., Di Leva, F., Bortolozzi, M., Ortolano, S., Donaudy, F., Petrillo, M., Melchionda, S., Lelli, A., Domi, T., Fedrizzi, L., Lim, D., Shull, G.E., Gasparini, P., Brini, M., Mammano, F., and Carafoli, E. 2007. A functional study of plasma-membrane calcium-pump isoform 2 mutants causing digenic deafness. Proc. Natl.Acad.Sci.U.S.A. 104: 1516-1521.

7. Friedman, T.B. and Griffith, A.J. 2003. Human nonsyndromic sensorineural deafness. Annu. Rev. Genomics Hum. Genet. 4: 341-402.

8. He, F., Wang, Z., Zhao, J., Bao, J., Ding, J., Ruan, H., Xie, Q., Zhang, Z., and Gao, X. 2003. Large scale screening of disease model through ENU mutagenesis in mice. Chin. Sci. Bull. 48: 2665-2671.

9. Kozel, P.J., Friedman, R.A., Erway, L.C., Yamoah, E.N., Liu, L.H., Riddle, T., Duffy, J.J., Doetschman, T., Miller, M.L., Cardell, E.L., and Shull, G.E. 1998. Balance and hearing deficits in mice with a null mutation in the gene encoding plasma membrane $\mathrm{Ca}^{2+}$-ATPase isoform 2.J. Biol. Chem. 273: 18693-18696.

10. McCullough, B.J., Adams, J.C., Shilling, D.J., Feeney, M.P., Sie, K.C., and Tempel, B.L. 2007.3p-Syndrome defines a hearing loss locus in 3p25.3. Hear. Res. 224: 51-60.

11. McCullough,B.J. and Tempel, B.L. 2004. Haplo-insufficiency revealed in deafwaddler mice when tested for hearing loss and ataxia. Hear. Res. 195: 90-102.

12. Penheiter, A.R., Filoteo, A.G., Croy, C.L., and Penniston, J.T. 2001. Characterization of the deafwaddler mutant of the rat plasma membrane calcium-ATPase 2. Hear. Res. 162: 19-28.
13. Schultz, J.M., Yang, Y., Caride, A.J., Filoteo, A.G., Penheiter, A.R., Lagziel, A., Morell, R.J., Mohiddin, S.A., Fananapazir, L., Madeo, A.C., Penniston, J.T., and Griffith, A.J. 2005. Modification of human hearing loss by plasma-membrane calcium pump PMCA2. N. Engl. J. Med. 352: 1557-1564.

14. Silverstein, R.S. and Tempel, B.L. 2006. Atp2b2, encoding plasma membrane $\mathrm{Ca}^{2+}$-ATPase type 2, (PMCA2) exhibits tissue-specific first exon usage in hair cells, neurons, and mammary glands of mice. Neuroscience 141: 245-257.

15. Spiden, S.L., Bortolozzi, M., Di Leva, F., de Angelis, M.H., Fuchs, H., Lim, D., Ortolano, S., Ingham, N.J., Brini, M., Carafoli, E., Mammano, F., and Steel, K.P. 2008. The novel mouse mutation Oblivion inactivates the PMCA2 pump and causes progressive hearing loss. PLoS Genet. 4: e1000238.

16. Street, V.A., McKee-Johnson, J.W., Fonseca, R.C., Tempel, B.L., and Noben-Trauth, K. 1998. Mutations in a plasma membrane $\mathrm{Ca}^{2+}$-ATPase gene cause deafness in deafwaddler mice. Nat. Genet. 19: 390-394.

17. Strehler, E.E. and Zacharias, D.A. 2001. Role of alternative splicing in generating isoform diversity among plasma membrane calcium pumps. Physiol. Rev. 81:21-50.

18. Sweadner, K.J. and Donnet, C. 2001. Structural similarities of $\mathrm{Na}, \mathrm{K}$-ATPase and SERCA, the $\mathrm{Ca}^{(2+)}$-ATPase of the sarcoplasmic reticulum. Biochem. J. 356: 685-704.

19. Takahashi, K. and Kitamura, K. 1999. A point mutation in a plasma membrane $\mathrm{Ca}^{(2+)}$-ATPase gene causes deafness in Wriggle Mouse Sagami. Biochem. Biophys. Res. Commun. 261: 773-778.

20. Tsai, Y.S., Pendse, A., Moy, S.S., Mohri, I., Perez, A., Crawley, J.N., Suzuki, K., and Maeda, N. 2006. A de novo deafwaddler mutation of Pmca2 arising in ES cells and hitchhiking with a targeted modification of the Pparg gene. Mamm. Genome 17: 716-722.

21. Van Laer, L., Cryns, K., Smith, R.J., and Van Camp, G. 2003. Nonsyndromic hearing loss. Ear Hear. 24: 275-288.

22. Willems, P.J. 2000. Genetic causes of hearing loss. N. Engl. J. Med. 342: 1101-1109.

23. Xiong, X., Qi, X., Ge, X., Gu, P., Zhao, J., Zhao, Q., and Gao, X. 2008. A novel Phex mutation with defective glycosylation causes hypophosphatemia and rickets in mice. J. Biomed. Sci. 15: 47-59.

24. Yamoah, E.N., Lumpkin, E.A., Dumont, R.A., Smith, P.J., Hudspeth, A.J., and Gillespie, P.G. 1998. Plasma membrane $\mathrm{Ca}^{2+}$-ATPase extrudes $\mathrm{Ca}^{2+}$ from hair cell stereocilia. $J$. Neurosci. 18: 610-624. 